\title{
Wave optics in spacetimes with compact gravitating object
}

\author{
Yasusada Nambu* \\ Department of Physics, Graduate School of Science, \\ Nagoya University, Chikusa, Nagoya 464-8602, Japan \\ Sousuke Noda \\ Center for Gravitation and Cosmology, \\ College of Physical Science and Technology, \\ Yangzhou University, \\ Yangzhou 225009, China \\ and \\ Yukawa Institute for Theoretical Physics, \\ Kyoto University, \\ Kitashirakawa Oiwakecho Sakyoku, \\ Kyoto 606-8502, Japan \\ Yuichiro Sakai \\ Department of Physics, Graduate School of Science, \\ Nagoya University, Chikusa-ku, Nagoya 464-8602, Japan
}

(Dated: September 17, 2019)

\begin{abstract}
We investigate the wave optics in spherically symmetric spacetimes: Schwarzschild black hole, spherical star with a perfect absorbing surface, and massless/massive Ellis wormholes. Assuming a point wave source, wave patterns and power spectrums for scattering waves are obtained by solving the scalar wave equation numerically. We found that the power spectrum at the observer in the forward direction shows oscillations with two characteristic periods determined by the interference effect associated with the photon sphere and the diffraction effect due to the absorbing boundary condition inside of the photon sphere.
\end{abstract}

PACS numbers: 04.20.-q, 04.70.-s, 42.25.Fx

Keywords: black hole; worm hole; wave optics; photon sphere; interference; diffraction

*Electronic address: nambu@gravity.phys.nagoya-u.ac.jp

$\dagger$ Electronic address: sousuke.noda@yukawa.kyoto-u.ac.jp 


\section{Contents}

I. Introduction 3

II. Wave optics in static spherically symmetric spacetimes 5

A. Wave equation with a point source

B. Our numerical methods

\begin{tabular}{lr} 
III. Results & 7 \\
\hline
\end{tabular}

A. Black hole case 8

B. Star case

C. Wormhole case

IV. Interpretation of power spectrum oscillations

\begin{tabular}{lr} 
V. Summary and conclusion & 20 \\
\hline
\end{tabular}

\begin{tabular}{ll} 
Acknowledgments & 20 \\
\hline
\end{tabular}

A. Wormhole spacetimes

References 


\section{INTRODUCTION}

The photon sphere is a set of circular unstable photon orbits around a gravitating object and it forms a two-dimensional sphere with a constant radius for spherically symmetric static spacetimes. Recently, related to the existence of the photon sphere, bright ring and the "shadow" of M87 have been observed [1. The properties of shadows of strong gravitating objects such as black holes have been studied in detail [2 10]. The shadow is a dark region on the observer's sky and its rim corresponds to the photon sphere projected onto the observer's sky. By its definition, information inside of the photon sphere cannot be detectable by light rays unless an illuminating light source is placed inside of the photon sphere.

Although the photon sphere is introduced in terms of null geodesics, which are rays in the geometrical optics, the relation to the quasinormal modes of the black hole has been also discussed so far [11]. The quasinormal modes of black holes are obtained as poles of the scattering matrix in the complex frequency domain and its eikonal limit corresponds to light rays of the unstable photon orbits around black holes. Based on the established treatment of wave scattering problems (partial wave decomposition, phase shift etc.; see Refs. [12, 13]), the photon sphere is related to Regge poles which are poles of the scattering matrix in the complex angular momentum space. Thus it is possible to understand properties of spacetimes with strong gravitating objects using wave optics. As an application to this direction, imaging of black hole photon sphere with waves was investigated by Kanai and Nambu [14] and Nambu and Noda [15]. The reconstruction of black hole images from scattering waves was attempted by Fourier transform of scattered waves. Other approaches to the wave scattering by black holes such as the evaluation of the differential cross section have been investigated by many authors [16 29]. Recently, wave scattering by stars is also discussed [30 32].

Concerning the wave optical effect for the weak gravitational lensing, interference fringe patterns in the spatial domain (scattering amplitude) and the frequency domain (power spectrum) are expected. They are caused by interference between two coherent light rays (direct rays). For the gravitational lensing by a black hole, an additional interference effect associated with the photon sphere is expected. Light rays can go around the black hole an arbitrary number of times (orbiting), the direct rays and these winding rays can interfere, and an additional component of fringe appears in the power spectrum. In the paper [15], the analytic expression for scattering waves by the Schwarzschild black hole was derived in the eikonal limit (the leading order of the wave effect), and wave optical images of Einstein rings and the photon sphere were obtained. Moreover, modulation of power spectrums caused by the photon sphere was also clarified. As an astrophysical application of wave optics to gravitational lensing systems, Yoo et al. [33] investigated the behavior of power spectrums from a point source and discussed the possibility to distinguish Ellis wormhole spacetimes from spacetimes with a point mass. Their analysis is based on the weak field approximation of the gravitational field (weak lensing effect). They concluded that the Ellis wormhole spacetime shows different behaviors of the power spectrum due to the $r^{-2}$ law of the wormhole's gravitational potential. However, their analysis lacks strong lensing effect associated with the photon sphere.

In this paper, we consider wave optical properties of spacetimes with the photon sphere. Let us consider a situation where a wave source is located outside of the photon sphere of the gravitating object and an observer detects a scattered signal. In the geometrical optics,

as light rays captured by the photon sphere cannot escape from it, we cannot look inside of 
the photon sphere using a light source placed outside of the photon sphere, if objects inside of the photon sphere do not emit and reflect light rays. However, in the wave optics, even if a part of the wave propagates inside of the photon sphere, it can escape to outside due to the wave effect and it is possible to extract information of interior of the photon sphere. This expectation is directly connected to the discrimination problem of gravitating objects called black hole mimickers such as ultracompact objects using wave optical effects. The black hole mimickers have photon spheres but no event horizons. Thus it is not possible to discriminate between the black hole mimickers and black holes using light rays. In this paper, as models of the black hole mimickers, we consider a spherical star with a perfect absorbing surface and massless/massive Ellis wormholes. We mainly focus on behavior of power spectrums in the forward scattering case; the path difference between two direct rays is zero and if the gravitating object has no structure like the photon sphere, we do not have any interference fringe in the power spectrum. However, if the gravitating object has the photon sphere or some structures, modulations of the power spectrum caused by interference between direct rays and orbiting rays are expected.

The structure of the paper is as follows. In Sec. II, we introduce our setup of the wave scattering problem in spherically symmetric spacetimes and explain our numerical methods. We present our numerical results in Sec. III for the Schwarzschild spacetime and the Ellis wormhole spacetime. In Sec. IV, we apply a formula in the wave optics to explain the interference fringe that appeared in power spectrums. Section V is devoted to the summary and conclusion. 


\section{WAVE OPTICS IN STATIC SPHERICALLY SYMMETRIC SPACETIMES}

In this section, we introduce the setup of the problem and numerical method to obtain scattering waves by a spherical gravitating object.

\section{A. Wave equation with a point source}

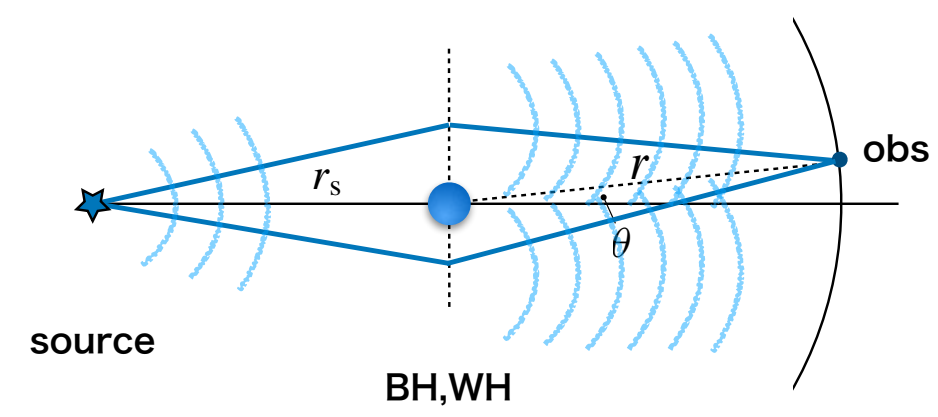

FIG. 1: Configuration of our wave scattering problem. An observer receives waves from a point wave source.

Figure 1 shows our setup of the wave scattering problem. We consider a massless scalar field as the benchmark treatment for wave scattering problems and we do not consider polarization degrees of freedom that are necessary for the electromagnetic and gravitational waves. The background geometry is assumed to be static and spherically symmetric spacetimes with the metric

$$
d s^{2}=g_{\mu \nu} d x^{\mu} d x^{\nu}=-f(r) d t^{2}+\frac{d r^{2}}{h(r)}+r^{2} d \Omega^{2} .
$$

For a monochromatic stationary wave with time dependence $e^{-i \omega t}$, the wave equation for the massless scalar field $\Phi$ reduces to the following Helmholtz type equation with a source term

$$
-g^{00} \omega^{2} \Phi+\frac{1}{\sqrt{-g}} \partial_{j}\left(\sqrt{-g} g^{j k} \partial_{k} \Phi\right)=-S_{\omega} \delta^{3}\left(\vec{r}, \vec{r}_{s}\right), \quad i, j, k=r, \theta, \phi,
$$

where a point wave source is placed at $\vec{r}_{s}$ and $\delta^{3}\left(\vec{r}, \vec{r}_{s}\right)$ is the invariant delta function $\frac{1}{\sqrt{-g}} \delta^{3}\left(\vec{r}-\vec{r}_{s}\right) . S_{\omega}$ denotes the Fourier amplitude of the wave source. In this paper, we assume the spectrum of the wave source has no $\omega$ dependence and $S_{\omega}$ is $\omega$ independent constant. The power spectrum of the wave at observing point is given by $|\Phi(\omega)|_{\text {obs. }}^{2} \cdot{ }^{1}$ We assume the wave source is placed on the $-z$ axis: $r=r_{s}, \theta=\pi$. That is $\delta^{3}\left(\vec{r}, \overrightarrow{r_{s}}\right) \propto \delta\left(r-r_{s}\right) \delta(\cos \theta+1)$. Owing to the symmetry of the spacetime, the wave function can be separated as

$$
\Phi(r, \theta)=\frac{1}{r} \sum_{\ell=0}^{\infty} R_{\ell}(r) P_{\ell}(\cos \theta),
$$

\footnotetext{
${ }^{1}$ Strictly speaking, $|\Phi(\omega)|^{2}$ represents energy of the wave per unit interval of the frequency and the power of the wave for interval of the frequency $\Delta \omega$ is represented as $|\Phi(\omega)|^{2} \Delta \omega$. In this paper, we call $|\Phi(\omega)|^{2}$ as the power spectrum for simplicity.
} 
and using the formula $\delta(\cos \theta+1)=\sum_{\ell=0}^{\infty}(-)^{\ell}(\ell+1 / 2) P_{\ell}(\cos \theta)$, the radial wave function $R_{\ell}$ obeys the following Schrödinger type equation

$$
\frac{d^{2} R_{\ell}}{d x_{\mathrm{tot}}^{2}}+\left(\omega^{2}-V_{\mathrm{eff}}\right) R_{\ell}=a_{s}(-)^{\ell}(\ell+1 / 2) \delta\left(r-r_{s}\right),
$$

where $a_{s}$ denotes the $\omega$ independent amplitude of the point source, the tortoise coordinate is introduced as

$$
x_{\mathrm{tot}}=\int \frac{d r}{\sqrt{f h}}
$$

and the effective potential is defined by

$$
V_{\text {eff }}=f \frac{\ell(\ell+1)}{r^{2}}+\frac{(f h)_{, r}}{2 r} .
$$

In this paper, we consider the Schwarzschild spacetime and the Ellis wormhole spacetime. The metric of the Schwarzschild spacetime with the tortoise coordinate $x_{\text {tot }}$ is

$$
\begin{aligned}
& d s_{\mathrm{Schw}}^{2}=\left(1-\frac{2 M}{r}\right)\left(-d t^{2}+d x_{\mathrm{tot}}^{2}\right)+r^{2} d \Omega^{2}, \\
& x_{\mathrm{tot}}=r+2 M \ln \left(\frac{r}{2 M}-1\right), \quad-\infty<x_{\mathrm{tot}}<+\infty .
\end{aligned}
$$

The effective potential is

$$
V_{\mathrm{eff}}=\left(1-\frac{2 M}{r}\right)\left(\frac{\ell(\ell+1)}{r^{2}}+\frac{2 M}{r^{3}}\right) .
$$

The metric of the Ellis wormhole (massless case) is

$$
\begin{aligned}
& d s_{\mathrm{WH}}^{2}=-d t^{2}+d x^{2}+r^{2} d \Omega^{2}, \\
& r=\sqrt{x^{2}+a^{2}}, \quad-\infty<x<+\infty,
\end{aligned}
$$

where the parameter $a$ represents the size of the wormhole's throat. The effective potential is

$$
V_{\mathrm{eff}}=\frac{\ell(\ell+1)}{r^{2}}+\frac{a^{2}}{r^{4}}
$$

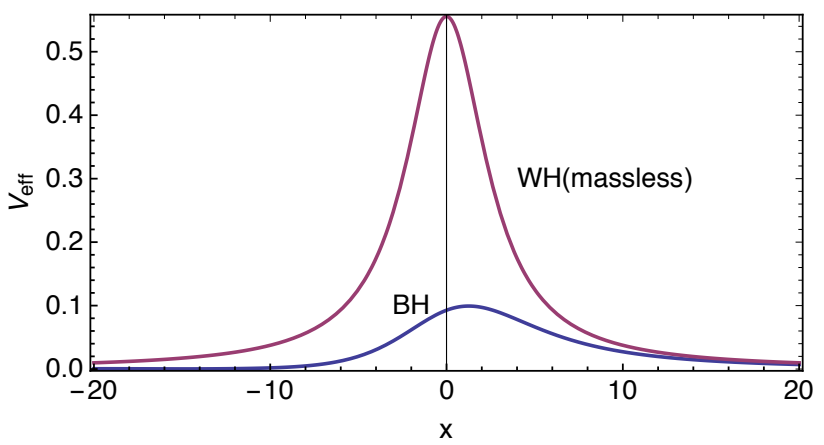

FIG. 2: Effective potentials for the Schwarzschild spacetime and the Ellis wormhole (massless) spacetime. The plot is with $\ell=2, a=3 M$. The circumference radius of the photon sphere for both spacetimes is $3 M$.

The metric for the massive Ellis wormhole is presented in Appendix A [Eq. (A2)]. Figure 2 shows these effective potentials. 


\section{B. Our numerical methods}

We present here the numerical method adopted in our analysis. We first obtain the solution of the radial equation (4) numerically. We impose two boundary conditions at $r=r_{\text {in }}$ (an inner boundary corresponds to the black hole horizon, the star's surface, and another asymptotic flat region of wormhole) and $r=r_{\text {out }}$ (an outer boundary corresponds to the spatially far region). We prepare two solutions of the homogeneous radial equation without a source term:

$$
\begin{array}{ll}
u_{1}(r), & r \in\left[r_{\text {in }}, r_{s}\right), \quad \text { BC is imposed at } r_{\text {in }}, \\
u_{2}(r), & r \in\left(r_{s}, r_{\text {out }}\right], \quad \text { BC is imposed at } r_{\text {out }}
\end{array}
$$

where $u_{1}$ is obtained by integrating the radial equation from $r_{\text {in }}$ to $r_{s}$, and $u_{2}$ is obtained by integrating the radial equation from $r_{\text {out }}$ to $r_{s}$.

Radial functions $u_{1}$ and $u_{2}$ do not satisfy the boundary condition at the source $r_{s}$ which is obtained by integrating (4) around $r_{s}$ :

$$
\left(\frac{d R_{2}}{d r}-\frac{d R_{1}}{d r}\right)_{r=r_{s}}=a_{s}(-)^{\ell}(\ell+1 / 2) \equiv \Delta_{\ell}, \quad\left(R_{2}-R_{1}\right)_{r=r_{s}}=0
$$

where $R_{1}(r)=R\left(r \leq r_{s}\right)$ and $R_{2}(r)=R\left(r \geq r_{s}\right)$. Using $u_{1}$ and $u_{2}$, we introduce new radial functions as

$$
R_{1}=c_{1} u_{1}, \quad R_{2}=c_{2} u_{2},
$$

where $c_{1}$ and $c_{2}$ are constants to be determined by the matching condition (13) at $r_{s}$ :

$$
c_{2} u_{2}^{\prime}\left(r_{s}\right)-c_{1} u_{1}^{\prime}\left(r_{s}\right)=\Delta_{\ell}, \quad c_{1} u_{1}\left(r_{s}\right)=c_{2} u_{2}\left(r_{s}\right) .
$$

We obtain

$$
c_{1}=\left(\frac{u_{2}}{W\left[u_{1}, u_{2}\right]}\right)_{r=r_{s}} \Delta_{\ell}, \quad c_{2}=\left(\frac{u_{1}}{W\left[u_{1}, u_{2}\right]}\right)_{r=r_{s}} \Delta_{\ell} .
$$

where $W=u_{1} u_{2}^{\prime}-u_{2} u_{1}^{\prime}$ is the Wronskian. Thus, $R_{1}$ and $R_{2}$ with required boundary conditions are

$$
R_{1}=\frac{\Delta_{\ell}}{W\left[u_{1}, u_{2}\right]_{r_{s}}} u_{1}(r) u_{2}\left(r_{s}\right), \quad R_{2}=\frac{\Delta_{\ell}}{W\left[u_{1}, u_{2}\right]_{r_{s}}} u_{1}\left(r_{s}\right) u_{2}(r)
$$

To obtain numerical solutions $u_{1}$ and $u_{2}$, we adopted the fourth-order Runge-Kutta method. We obtained the radial wave function within the relative errors $10^{-5}$. Concerning the value $\ell_{\max }$ for the summation of the partial waves (3), we determined it by checking the convergence of $\Phi$ at $r_{\mathrm{obs}}=20 M, \theta=0$. For $M \omega<1$, we obtained $\ell_{\max }=10-13$. For $1<M \omega \leq 10$, we found that $\ell_{\max }=9 \times(M \omega+1)$ for the black hole cases. For calculations with stars and a wormhole, we determined $\ell_{\max }$ using the same method.

\section{RESULTS}

We obtained the scattering wave from a monochromatic point wave source for the Schwarzschild spacetime (black hole, stars with a perfect absorbing surface) and the Ellis wormhole spacetime. The point wave source is placed at $r_{s}=6 M, \theta=\pi$ with frequencies $0<M \omega \leq 10$. The observing point of power spectrum is $r_{\mathrm{obs}}=20 M$. 


\section{A. Black hole case}

Numerical results for the Schwarzschild spacetime are as follows.

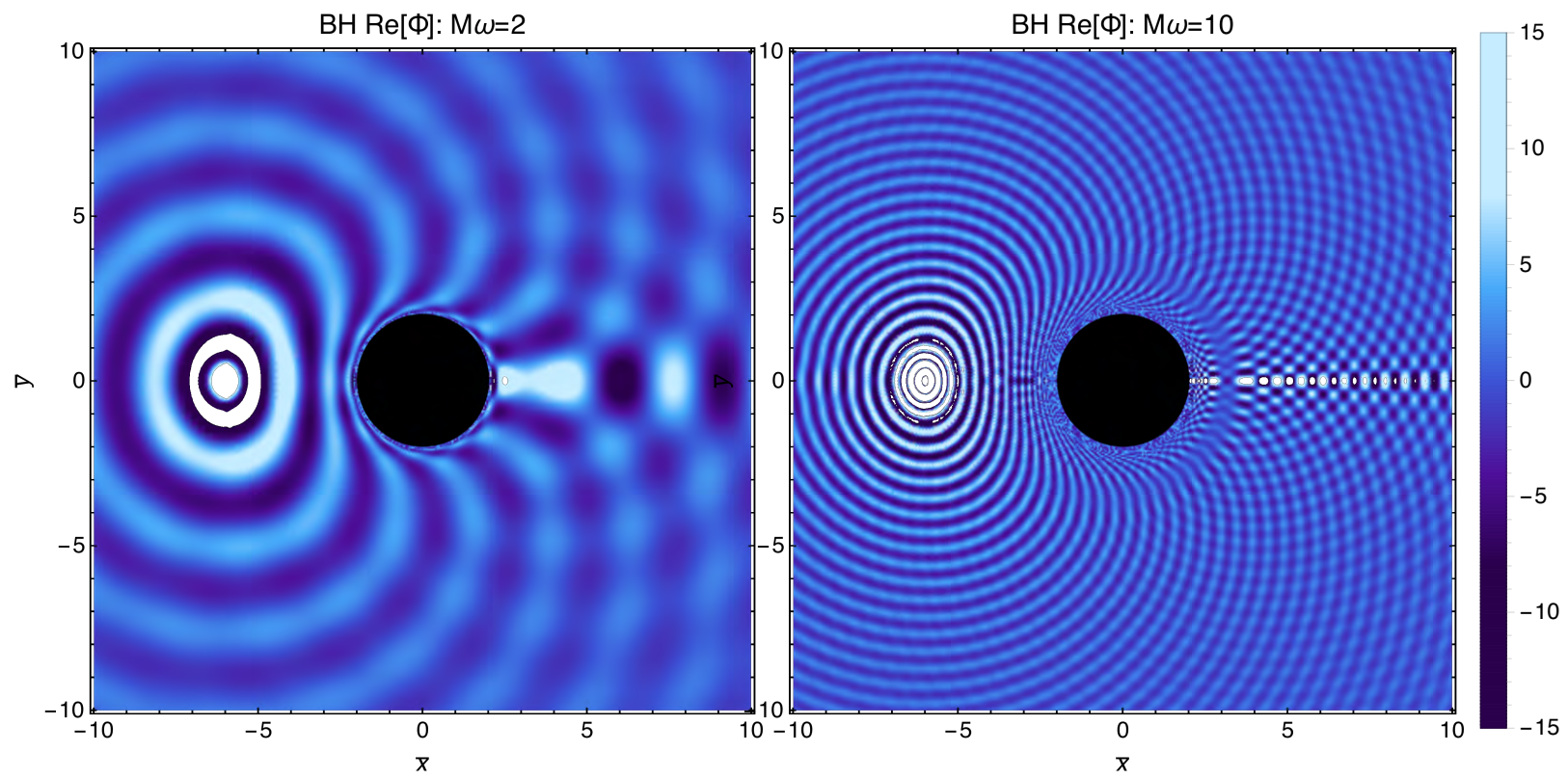

FIG. 3: Real part of $\Phi$ for $M \omega=2,10$. The Cartesian coordinates $\bar{x}$ and $\bar{y}$ are introduced by $\bar{x}=r \cos \theta, \bar{y}=r \sin \theta$.

Figure 3 shows the real part of scattering waves. For $M \omega=10$, we can see the circlelike wave pattern corresponding to the photon sphere at $r \simeq 3 M$ and the bright line (caustics) behind the black hole, while, for the $M \omega=2$ case, these features are blurred due to the wave effect. Figures 4, 5 and 6] show the intensity of scattered waves at $r_{\text {obs }}$. They show interference fringes in both the spatial domain $(\theta)$ and the frequency domain $(\omega)$. Namely, $|\Phi|^{2}$ on a constant- $\theta$ slice is the power spectrum at the observing point and $|\Phi|^{2}$ on a constant- $M \omega$ slice represents the scattering amplitude for a fixed frequency. 


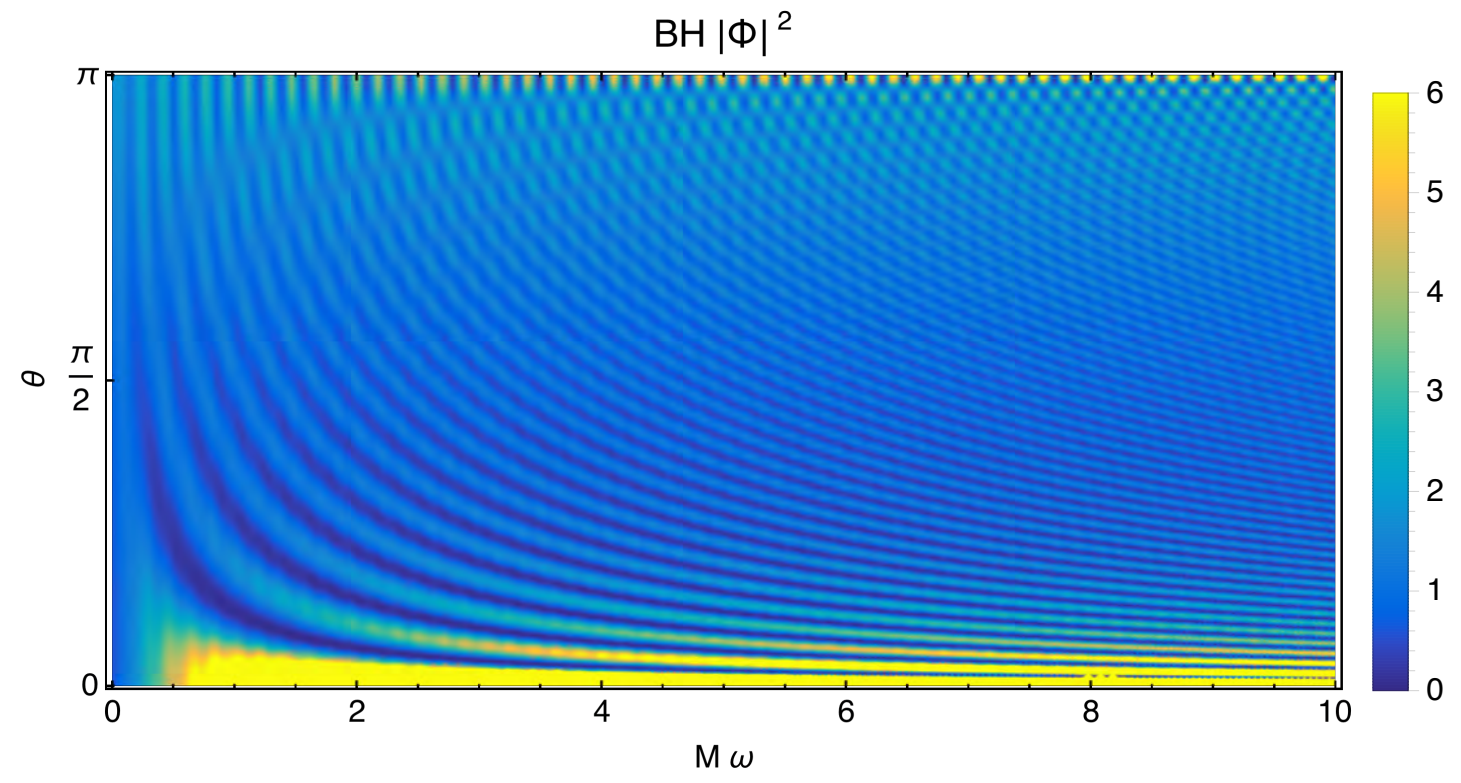

FIG. 4: $|\Phi|^{2}$ at $r_{\text {obs }}$ as a function of $(\omega, \theta)$. Interference fringe appears in the two-dimensional parameter space.
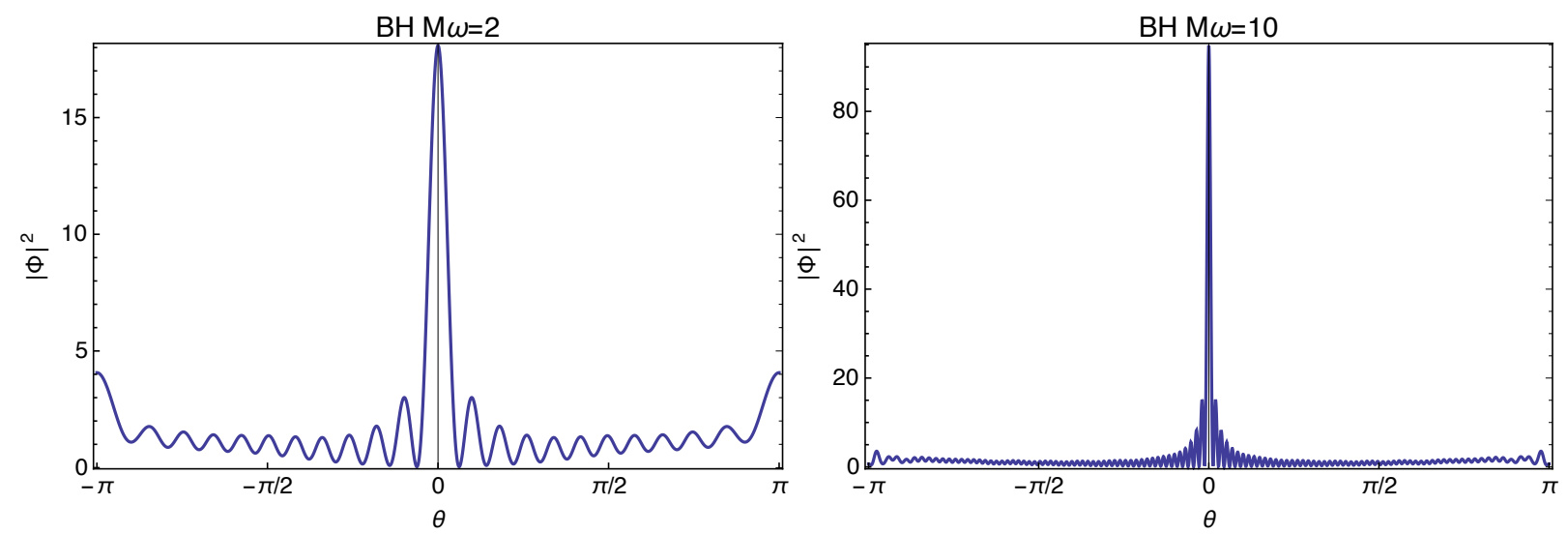

FIG. 5: Sections of $M \omega=2$ and $M \omega=10$ of Fig. 4 (scattering amplitudes). These plots show interference fringes in the spatial domain. 

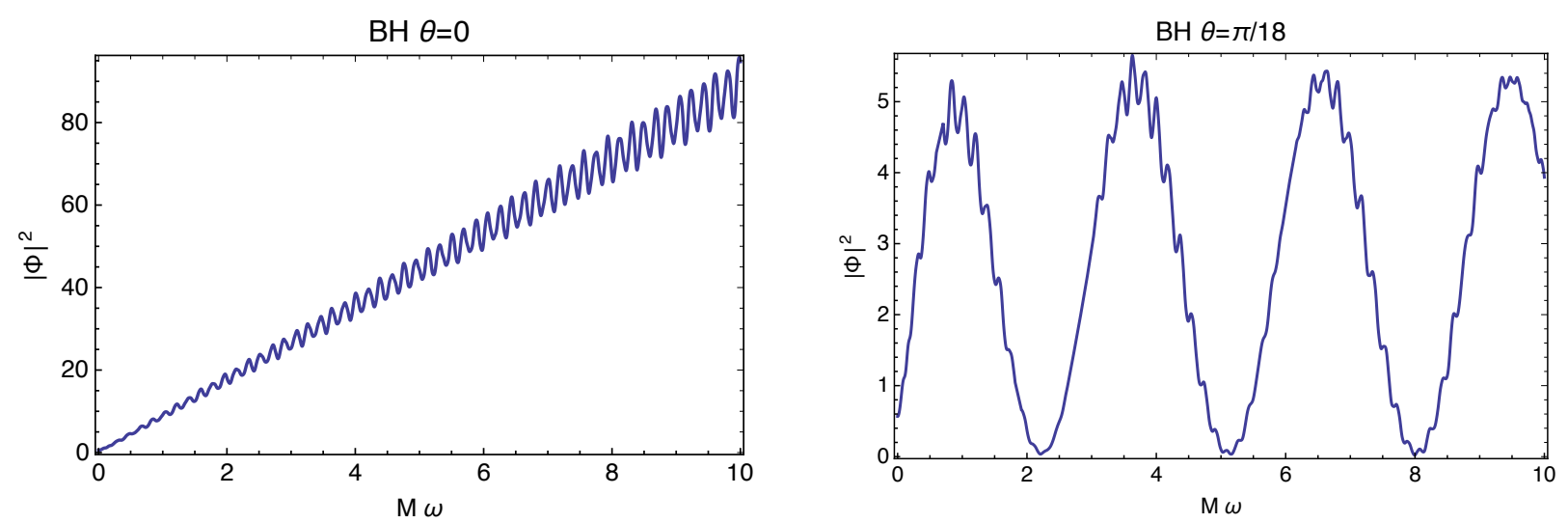

FIG. 6: Sections of $\theta=0$ and $\theta=\pi / 18$ of Fig. 4 (power spectrums). These plots show interference fringes in the frequency domain.

We explain basic features of power spectrums (Fig. 6). We observe interference fringes in the power spectrums. For $\theta=\pi / 18$, we have two components of oscillations. The component with the longer period is originated from interference between two light rays traveling far from the black hole (direct rays) and is associated with the weak gravitational lensing effect. The period of this oscillation is proportial to inverse of path difference $(\propto 1 / \theta)$ for small $\theta$ and for $\theta=0$ limit, the period becomes infinite and we do not have oscillation in the power spectrum caused by the interference between direct rays. The other component of oscillation has a shorter period $M \Delta \omega \sim 0.2$ which is independent of the scattering angle $\theta$ and exists even for the forward direction $\theta=0$; in this case the path difference between two direct rays becomes zero and we cannot expect interference fringe in the power spectrum. Thus we conclude that this oscillation of the power spectrum in the forward direction is caused by interference between winding rays and direct rays, and is peculiar to spacetimes with an unstable photon orbit (photon sphere). As another feature, the power spectrum at $\theta=0$ increases with $M \omega$. This is related to the caustics where the scattered waves are focused and the intensity diverges in the geometrical optics limit $(M \omega \rightarrow \infty)$. The caustic points of a spherical lens are located right behind the lens object $(\theta=0$ line), and the sharpness of the divergence gets mild as the wavelength becomes large due to a wave effect. Therefore, we can see the feature in the left panel of Fig. 6 .

To clarify that the period $M \Delta \omega \sim 0.2$ corresponds to the scale of the unstable photon orbit, we consider a toy model of gravitational lensing by the black hole (Fig. 7). 


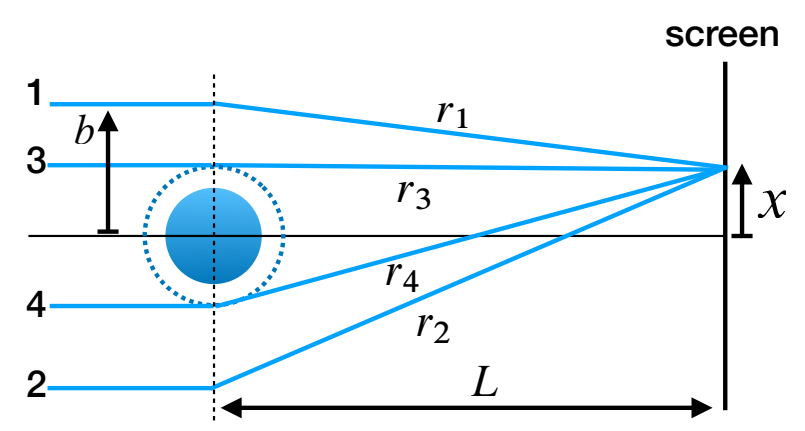

FIG. 7: A model of gravitational lensing by a black hole. Ray 1 and ray 2 represent direct rays of which the deflection angle is given by $\theta_{\text {defl }}$. Ray 3 and ray 4 represent winding rays.

We assume all rays follow straight lines as an approximation and the impact parameter $b$ is sufficiently smaller than $L$. Rays 1 and 2 are direct rays and their deflection angle is assumed to obey Einstein's formula

$$
\theta_{\text {defl }}=-\frac{4 M}{b}
$$

where $b$ denotes the impact parameter of each ray. The position $x$ on the screen and $b$ are related by

$$
b_{1,2}=\frac{x \pm \sqrt{x^{2}+16 L M}}{2} .
$$

Rays 3 and 4 correspond to winding rays with the impact parameter $3 \sqrt{3} M$. The path lengths of each ray are

$$
\begin{aligned}
& r_{1}=\sqrt{L^{2}+\left(b_{1}-x\right)^{2}}, \quad r_{2}=\sqrt{L^{2}+\left(b_{2}-x\right)^{2}}, \\
& r_{3}=\sqrt{L^{2}+\left(b_{3}-x\right)^{2}}+2 \pi \times\left|b_{3}\right|, \quad r_{4}=\sqrt{L^{2}+\left(b_{4}-x\right)^{2}}+2 \pi \times\left|b_{4}\right|,
\end{aligned}
$$

where $b_{3}=3 \sqrt{3} M, b_{4}=-3 \sqrt{3} M$. We assume winding rays go around the black hole one round. Then ignoring the difference of amplitudes for each ray, the wave on the screen is given by

$$
\Phi=e^{i \omega r_{1}}+e^{i \omega r_{2}}+c\left(e^{i \omega r_{3}}+e^{i \omega r_{4}}\right),
$$

where $c \approx 0.1$ represents the relative amplitude for winding rays but the value of this constant does not affect the period of interference in our estimation. For the forward direction $\theta=0(x=0)$, by neglecting $O(b / L)$ terms in the phase, we obtain

$$
|\Phi|^{2} / 4 \approx 1+c^{2}+2 c \cos [\omega(6 \pi \sqrt{3}-2) M]
$$

and the period of the power spectrum is given by

$$
M \Delta \omega=\frac{1}{3 \sqrt{3}-1 / \pi} \approx \frac{1}{3 \sqrt{3}} \approx 0.2
$$

This value is consistent with the period of oscillation observed in our numerical calculation. 


\section{B. Star case}

To clarify wave effects associated with the photon sphere, we investigate stars with a perfect absorbing surface in the Schwarzschild spacetime. We consider the following form of the effective potential:

$$
V(r)=V_{\mathrm{BH}}(r) \theta\left(r-r_{\mathrm{star}}\right),
$$

where $\theta(r)$ is the unit step function, $V_{\mathrm{BH}}(r)$ denotes the effective potential (9) of the Schwarzschild spacetime and $r_{\text {star }}$ is the radius of the star. This form of the potential models perfect absorptions of incoming waves at the surface of the star:

$$
R_{\ell}\left(x_{\mathrm{tot}}\right) \propto e^{-i \omega x_{\mathrm{tot}}} \text { for } r \leq r_{\mathrm{star}} .
$$

We consider four different values of radii $r_{\text {star }}=2.5 M, 3 M, 3.5 M, 4 M$. The obtained wave patterns in these models are shown in Fig. 8.
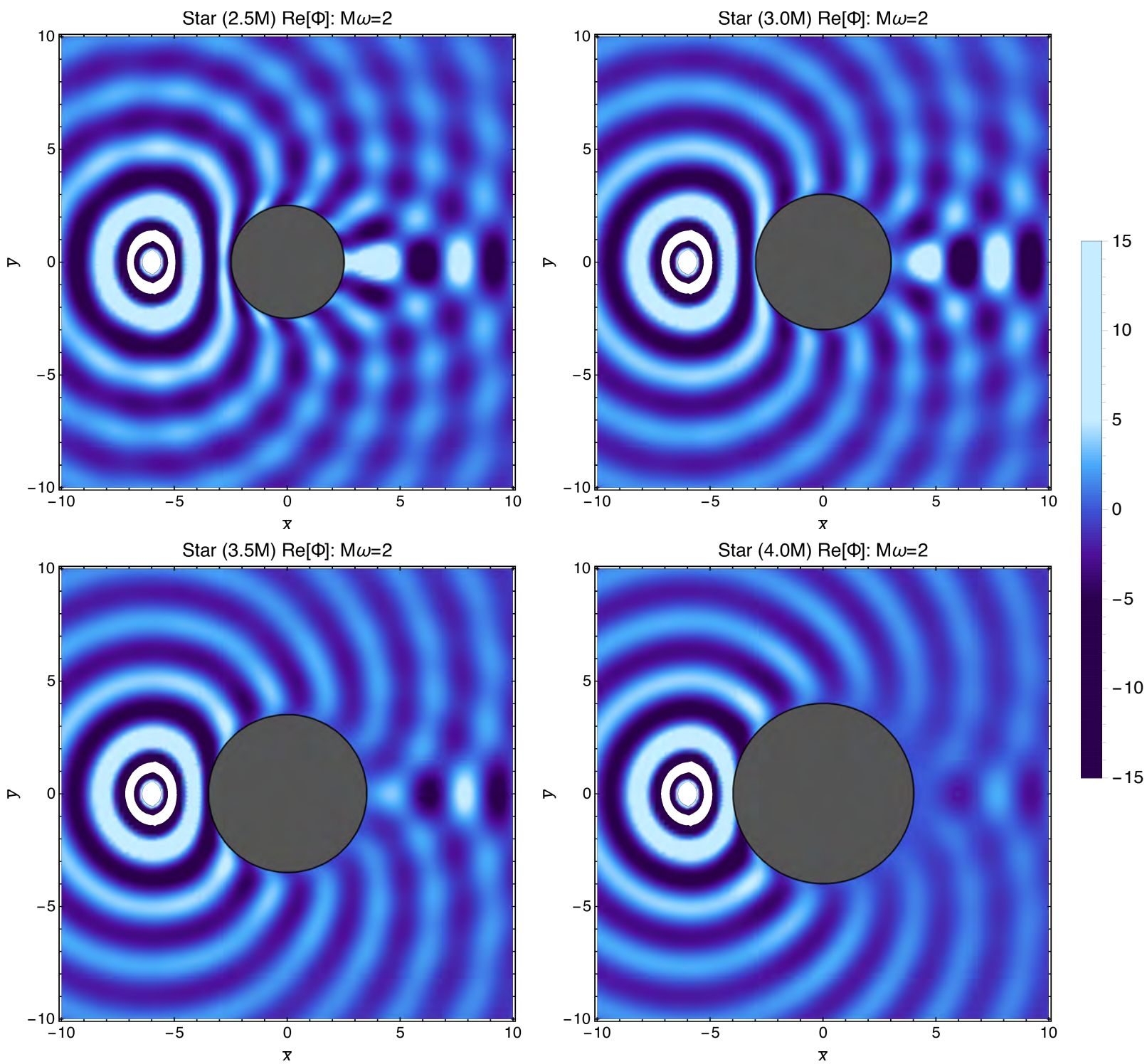

FIG. 8: Real part of $\Phi$ with $M \omega=2$ for stars with a perfect absorbing surface. Four panels correspond to different values of the radii of stars $2.5 M, 3 M, 3.5 M, 4 M$. 
The star with radius $2.5 \mathrm{M}$, which is smaller than the photon sphere of the Schwarzschild spacetime $3 M$, is a model of black hole mimickers (gravastar, boson star, etc.). For this case, the power spectrum shows oscillation with two different periods (the upper left panel in Fig. 9). The shorter one is $M \Delta \omega_{1} \sim 0.2$, exactly the same value as the black hole case, and is caused by interference between direct rays and winding rays associated with the photon sphere. In addition, oscillations with the longer period $M \Delta \omega_{2} \sim 2$ are superposed. We expect this component is due to the diffraction effect caused by the surface of the star. To justify this interpretation, we also investigated power spectrums for stars with other radii.
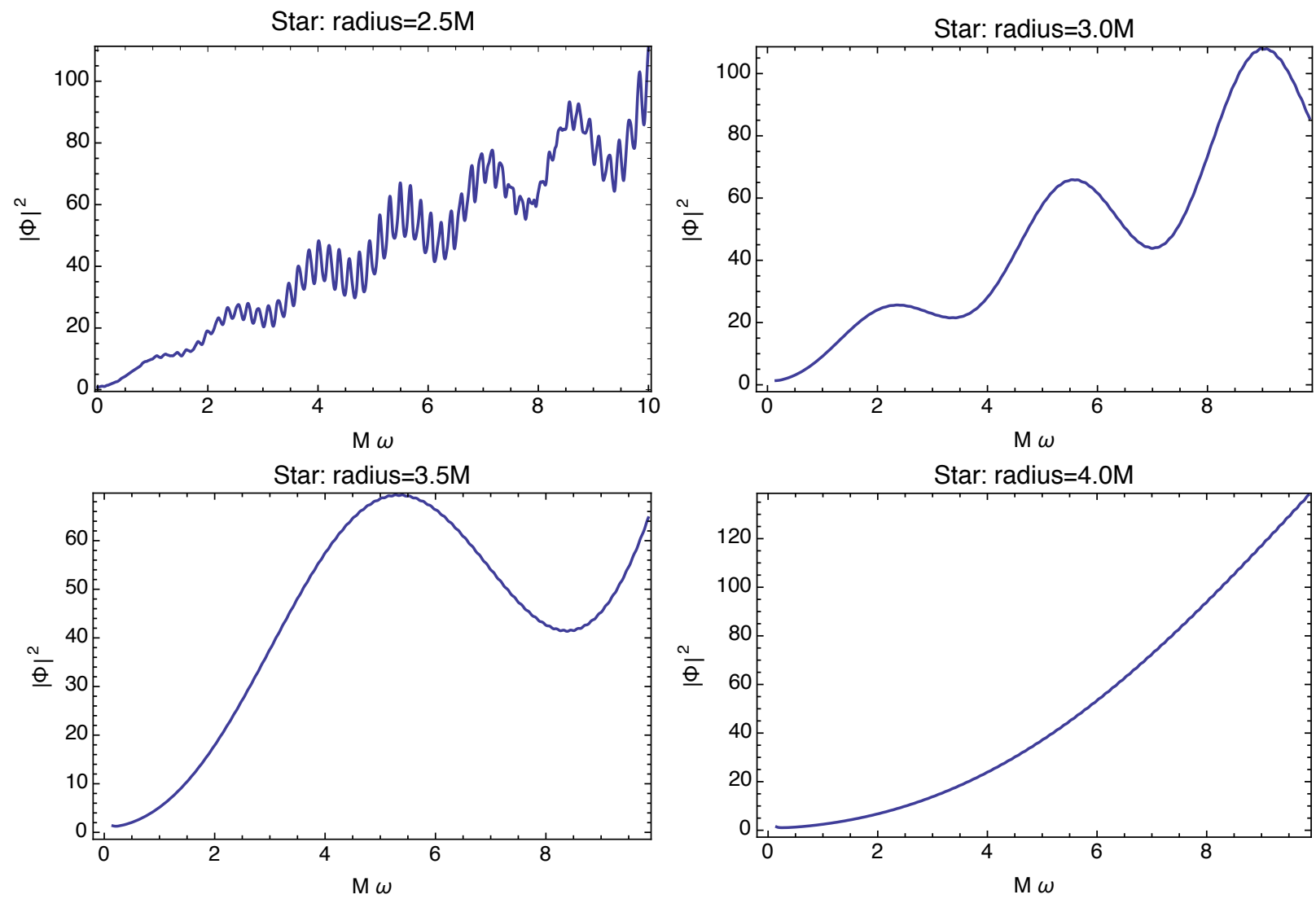

FIG. 9: The power spectrum in the forward direction for stars with different radii $2.5 M, 3 M, 3.5 M, 4 M$.

For stars with radius larger than $3 M$, the photon spheres are hidden by the surface of stars and we do not have oscillation with $M \Delta \omega_{1} \sim 0.2$. Power spectrums show oscillation with the longer period $M \Delta \omega>2$ depending on the radius of the stars. It is possible to estimate this period based on the diffraction effect of waves (see Sec. IV).

Figure 10 summarizes the behavior of power spectrums for the black hole case and stars with different radii cases. 

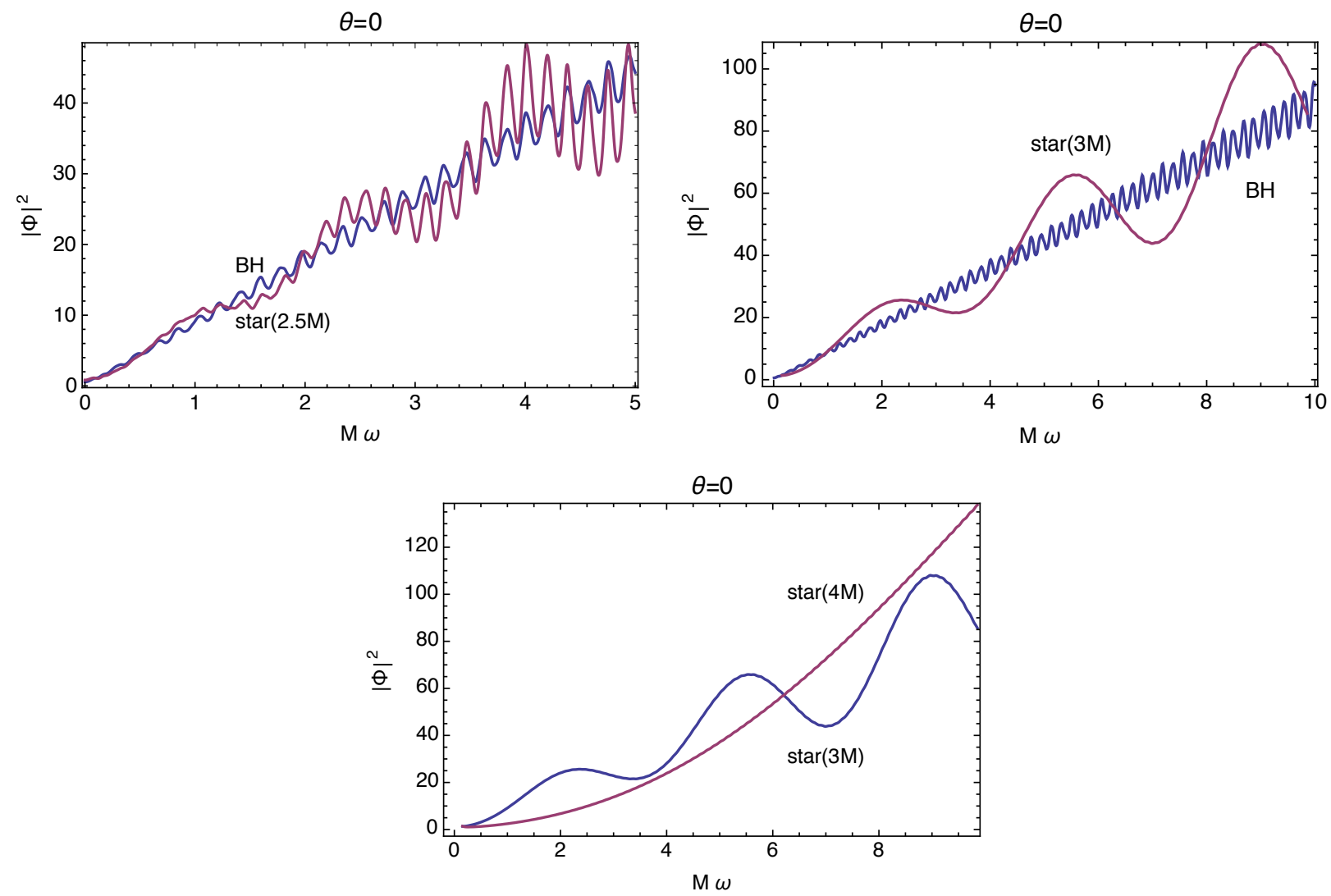

FIG. 10: Power spectrum at $r_{\text {obs }}$ for the black hole and stars with radii $2.5 M, 3 M, 3.5 M, 4 M$. The first panel is plotted in the range $0 \leq M \omega \leq 5$ to show the period of the oscillation clearly. 


\section{Wormhole case}

We present the numerical result for the Ellis wormhole spacetime.

a. Massless case. We choose parameters of the Ellis wormhole as $m=0, a=3 M$. In this case, the circumference radius of the wormhole throat and the photon sphere coincide. The obtained wave patterns are shown in Fig. 11.
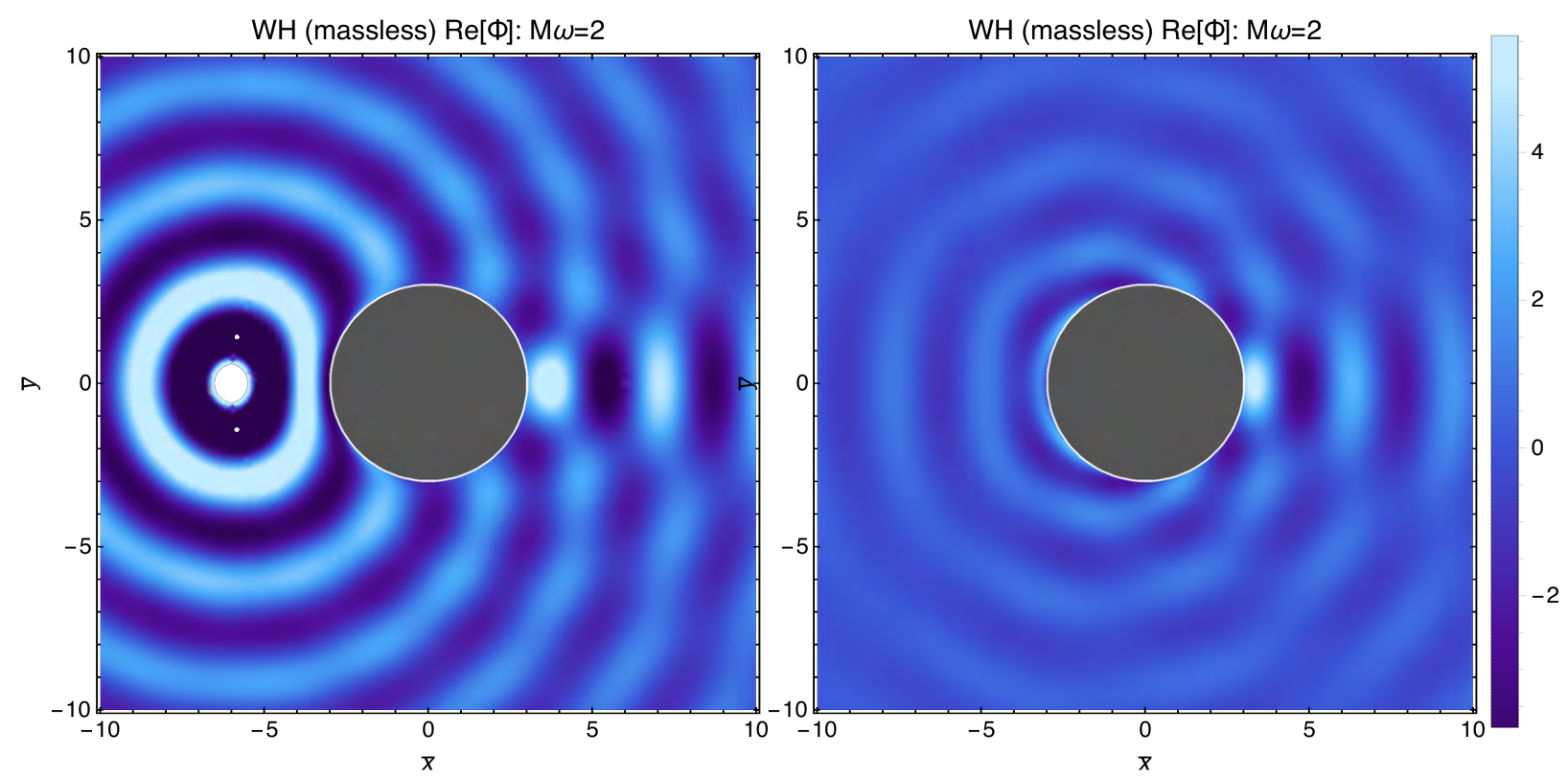

FIG. 11: The real part of $\Phi$ for the massless wormhole with $M \omega=2$. The left and right panels correspond to wormhole regions $x>0$ and $x<0$, respectively. The point wave source is placed in $x>0$ region. The coordinates $\bar{x}$ and $\bar{y}$ are introduced by $\bar{x}=\sqrt{x^{2}+a^{2}} \cos \theta, \bar{y}=\sqrt{x^{2}+a^{2}} \sin \theta$.

The power spectrum has the same behavior as that of the black hole (Fig. 12): it shows an oscillation with a period $M \Delta \omega \sim 0.4$.
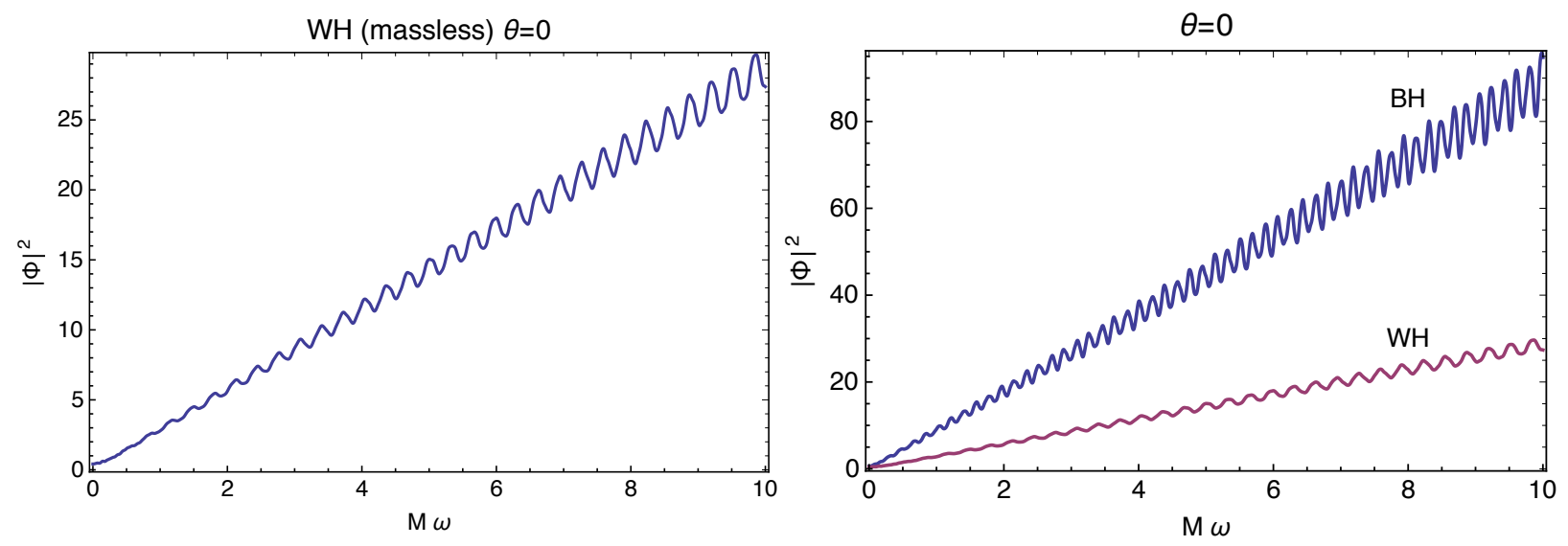

FIG. 12: Power spectrum at $r_{\text {obs }}$ for the massless Ellis wormhole. 
We can explain this value using the same toy model presented in Fig. 7. For the massless Ellis wormhole, the deflection angle is given by 34]

$$
\theta_{\mathrm{defl}}=\frac{\pi}{4}\left(\frac{a}{b}\right)^{2}
$$

Then the period of oscillation in the power spectrum becomes

$$
M \Delta \omega=M\left[a-\frac{1}{4}\left(\frac{a}{2 \pi L}\right)^{2 / 3}\right]^{-1} \sim 0.36
$$

for $a=3 M$ and $L=20 M$ and this formula well explains the value obtained by our numerical calculation. In this formula, the dependence of $L$ (distance from the observer to the wormhole) appears due to $b^{-2}$ behavior of the deflection angle, which is different from that for the black hole and stars.

b. Massive case. We choose the parameters of the wormhole as $m=M, a=$ $1.305716 M$. For these parameters, the size of the throat is $3 M$ and the photon sphere is $3.4823 M$.

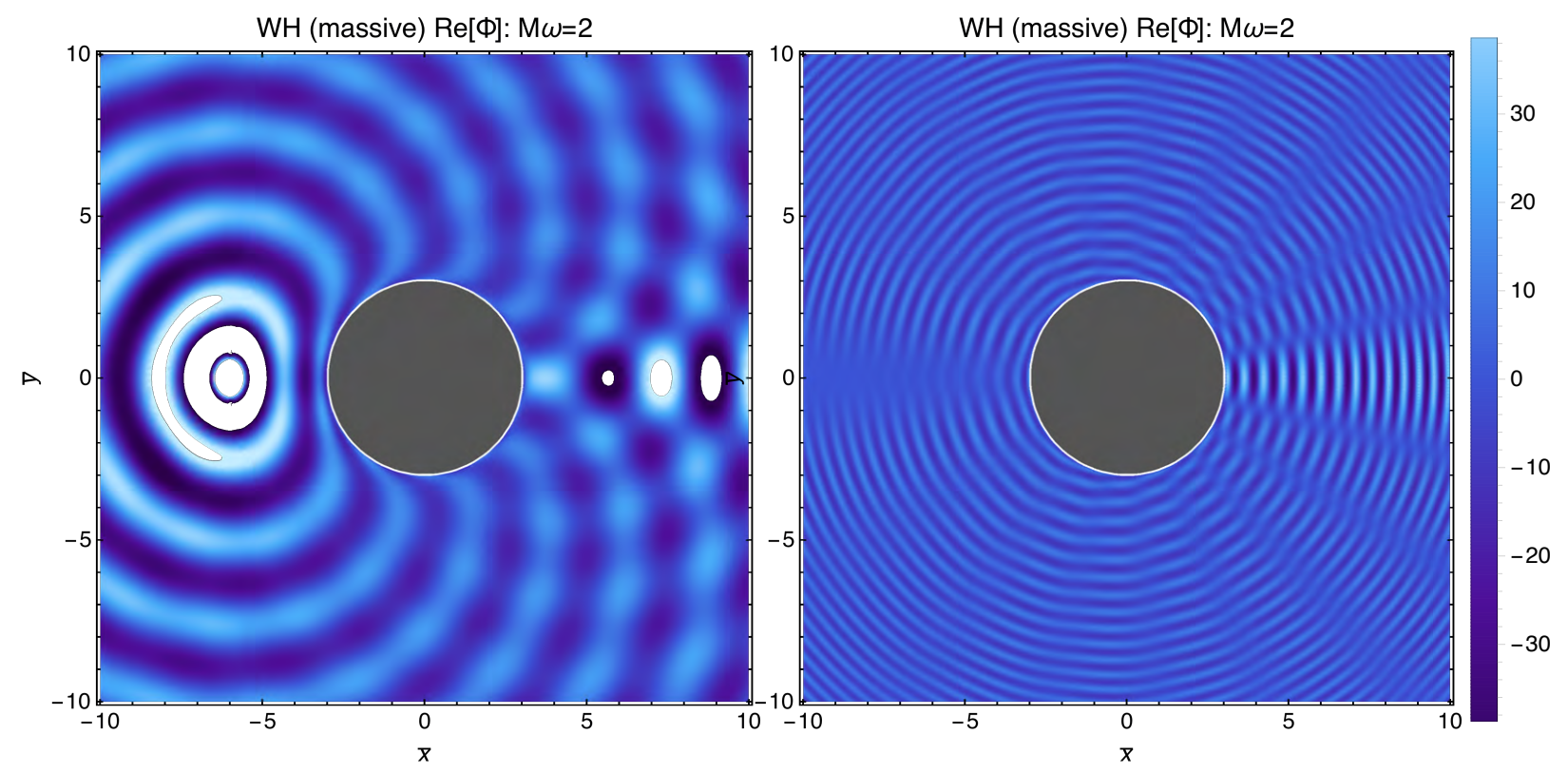

FIG. 13: The real part of $\Phi$ for the massive wormhole with $M \omega=2$. The left and right panels correspond to wormhole regions $x>0$ and $x<0$, respectively. The coordinates $\bar{x}$ and $\bar{y}$ are introduced as $\bar{x}=\sqrt{x^{2}+a^{2}-m^{2}} \cos \theta, \bar{y}=\sqrt{x^{2}+a^{2}-m^{2}} \sin \theta$.

We notice that intervals of the wave front are different for the $x>$ region and the $x<0$ region (Fig. 13). This is caused by different asymptotic behaviors of metric A2). For $x \rightarrow \infty$, the metric is

$$
d s^{2} \approx-\left(1-\frac{2 m}{r}\right) d t^{2}+\left(1+\frac{2 m}{r}\right) d r^{2}+r^{2} d \Omega^{2}
$$

whereas for $x \rightarrow-\infty$,

$$
d s^{2} \approx-\alpha^{-2}\left(1+\frac{2 \alpha m}{r}\right) d t^{2}+\left(1-\frac{2 \alpha m}{r}\right) d r^{2}+r^{2} d \Omega^{2}
$$


with $\alpha=\exp \left(\pi m / \sqrt{a^{2}-m^{2}}\right)$ and this metric represents a spacetime with negative gravitational mass $-\alpha m$. Let us consider the radial null vector $k_{\mu}=(\omega, k)$. Then $\omega$ and $k$ are connected by the relation

$$
k=\omega \sqrt{\left|\frac{g^{t t}}{g^{r r}}\right|}= \begin{cases}\omega(1+2 m / r) & \text { for } \quad x \rightarrow \infty \\ \omega \alpha(1-2 \alpha m / r) & \text { for } \quad x \rightarrow-\infty\end{cases}
$$

As the interval of wave front $\Delta r$ is determined by $k \Delta r=$ const, thus $\Delta r \propto 1 / k$. For $\alpha \gg 1$, which holds for values of present parameters, the interval of the wave front in the region $x<0$ becomes much smaller compared to that in the region $x>0$.

Figure 14 shows the power spectrum for the massive wormhole.
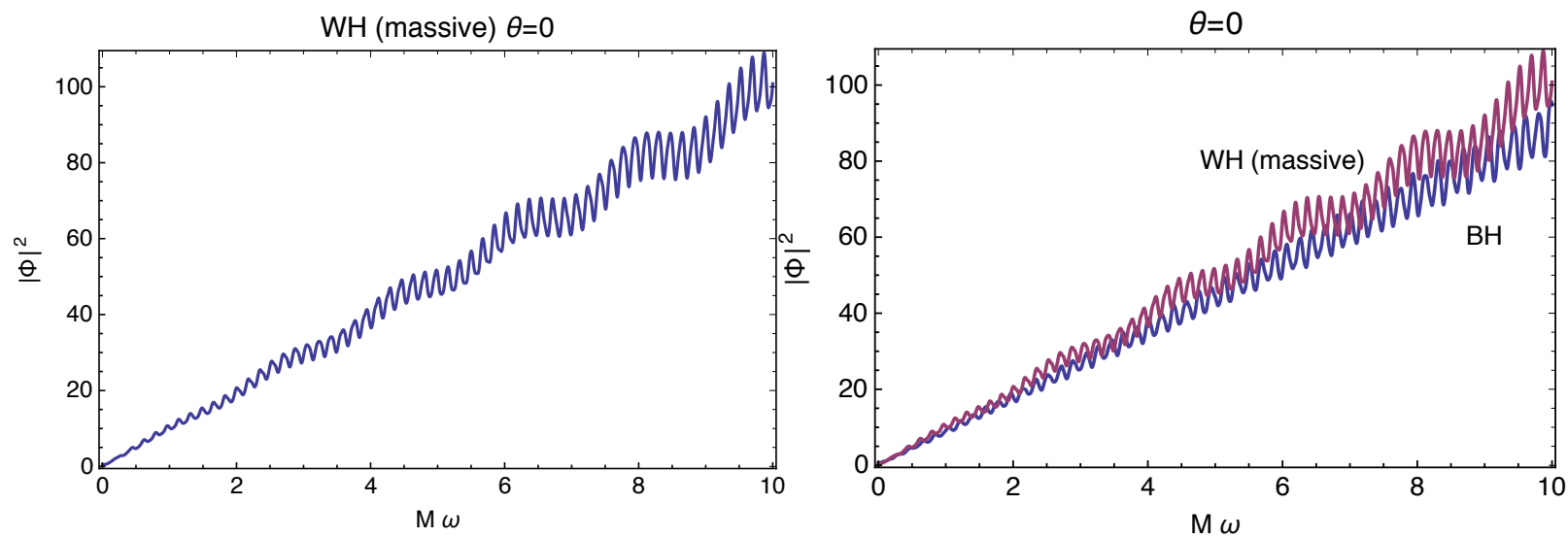

FIG. 14: Left: Power spectrums at $r_{\mathrm{obs}}$ for the massive Ellis wormhole. Right: Power spectrums for the black hole and the massive Ellis wormhole.

Power spectrums have oscillations with two different periods. The shorter one $M \Delta \omega_{1} \sim 0.2$ is due to interference between direct rays and winding rays, and the value coincides with that for the black hole because the radius of the photon sphere and the deflection angle for direct rays are the same as the black hole. The longer one $M \Delta \omega_{2} \sim 2$ comes from the diffraction effect by the absorbing region: for the massive wormhole, this region corresponds to the wormhole throat $3 M$ which is smaller than the radius of photon sphere $3.48 M$. 


\section{INTERPRETATION OF POWER SPECTRUM OSCILLATIONS}

In this section, we will provide theoretical justification for behaviors of the power spectrums based on analytic formulas of wave optics. We have two key factors associated with wave effects in our scattering problem: interference and diffraction.

At a distant observing point $r$, the scattering wave from a point wave source at $r_{s}$ is represented as [15]

$$
\Phi(r, \theta) \approx \frac{e^{i \omega\left(x+x_{s}\right)}}{4 \pi i \omega r r_{s}} \sum_{\ell=0}^{\infty} \lambda e^{i \frac{\lambda^{2}}{2 \omega \bar{r}}} e^{2 i \delta_{\ell}} P_{\ell}(\cos \theta), \quad \lambda:=\ell+\frac{1}{2},
$$

where $x$ and $x_{s}$ are tortoise coordinates corresponding to $r$ and $r_{s}$, and $\bar{r}=r r_{s} /\left(r+r_{s}\right) . \delta_{\ell}$ represents the phase shift. Applying Poisson's sum formula, we can replace the sum with respect to $\ell$ to the integral over continuous variable $\lambda$. In the eikonal limit, it can be shown that

$$
\Phi(r, \theta) \approx \frac{e^{i \omega\left(x+x_{s}\right)}}{4 \pi i \omega r r_{s}}\left[\int_{0}^{\infty} d \lambda \lambda e^{i \frac{\lambda^{2}}{2 \omega \bar{r}}} e^{2 i \delta_{\lambda-1 / 2}} J_{0}(\lambda \theta)+2 \pi i \sum_{n=0}^{\infty} \lambda_{n} \gamma_{n} e^{i \frac{\lambda_{n}^{2}}{2 \omega \bar{r}}} J_{0}\left(\lambda_{n} \theta\right) f\left(\lambda_{n}\right)\right]
$$

where $J_{0}$ is the Bessel function with the 0th order and

$$
\begin{aligned}
& \lambda_{n}=\lambda_{c}+i\left(n+\frac{1}{2}\right), \quad \lambda_{c}=3 \sqrt{3} M \omega \\
& \gamma_{n}=-\frac{i}{\sqrt{2 \pi}}\left(\frac{\lambda_{n}}{\lambda_{c}}\right)\left(n+\frac{1}{2}\right)^{n+1 / 2} \frac{e^{-(n+1 / 2)}}{n !}, \quad f\left(\lambda_{n}\right)=\frac{1}{1-e^{-2 i \pi\left(\lambda_{n}-1 / 2\right)}} .
\end{aligned}
$$

The first term in (33) corresponds to the Fresnel-Kirchhoff diffraction formula in the wave optics [35], and the second term comes from the contribution of poles in the $S$ matrix $e^{2 i \delta_{\ell}}$ in the complex $\ell$ plane (Regge poles). This term represents the orbiting effect associated with the photon sphere. After taking the $n$ sum, we obtain [15]

$$
\Phi(r, \theta) \propto \frac{1}{\omega} \int_{0}^{\infty} d \lambda \lambda e^{i \frac{\lambda^{2}}{2 \omega \bar{r}}} e^{2 i \delta_{\lambda-1 / 2}} J_{0}(\lambda \theta)+\frac{1}{\omega} \sqrt{\frac{\pi}{2}} e^{-\pi-i \pi / 4+i \pi \lambda_{c}} \lambda_{c} e^{\frac{i \lambda_{c}^{2}}{2 \omega \bar{r}}} \sqrt{\omega \bar{\omega}} J_{0}\left(\lambda_{c} \theta\right) .
$$

For the forward direction $\theta=0$,

$$
\begin{aligned}
\Phi & \propto \frac{1}{\omega} \int_{0}^{\infty} d \lambda \lambda e^{i \frac{\lambda^{2}}{2 \omega \bar{r}}} e^{2 i \delta_{\lambda-1 / 2}}+\frac{1}{\omega} \sqrt{\frac{\pi}{2}} e^{-\pi-i \pi / 4+i \pi \lambda_{c}} \lambda_{c} e^{\frac{i \lambda_{c}^{2}}{2 \omega \bar{r}}} \sqrt{\omega \bar{r}} \\
& =\omega \int_{b_{0}}^{\infty} d b b e^{i \frac{\omega b^{2}}{2 \bar{r}}} e^{2 i \delta_{b}}+\sqrt{\frac{\pi}{2}} e^{-\pi-i \pi / 4+i \pi \omega b_{c}} b_{c} e^{\frac{i \omega b_{c}^{2}}{2 \bar{r}}} \sqrt{\omega \bar{r}}
\end{aligned}
$$

where we introduced the impact parameter $b=\lambda / \omega$ and a lower cutoff $b_{0}$ of the integral, which represents the effect of a perfect absorbing region (the black hole horizon, surface of the stars). Concerning the form of the phase shift for direct rays, we adopt $\delta_{b}=-2 M \omega \ln (b \omega)$ which results in the scattering angle in the eikonal limit as

$$
2 \frac{d}{d(b \omega)} \delta_{b}=-\frac{4 M}{b}
$$


and reproduces Einstein's formula of deflection angle. Although this formula is correct only for rays with sufficiently large impact parameters compared to the size of the photon sphere, it is adequate for our purpose here to obtain a qualitative understanding of the oscillation of power spectrums. Then after performing the integral, the first term in Eq. (37) becomes

$$
\bar{r}(2 i \bar{r} \omega)^{-2 i M \omega}\left[2 M \omega \Gamma(-2 i M \omega)-i\left\{\Gamma(1-2 i M \omega)-\Gamma\left(1-2 i M \omega,-\frac{i b_{0}^{2} \omega}{2 \bar{r}}\right)\right\}\right]
$$

where the third term denotes the incomplete gamma function. We show the behavior of the obtained analytic formula in Fig. 15.
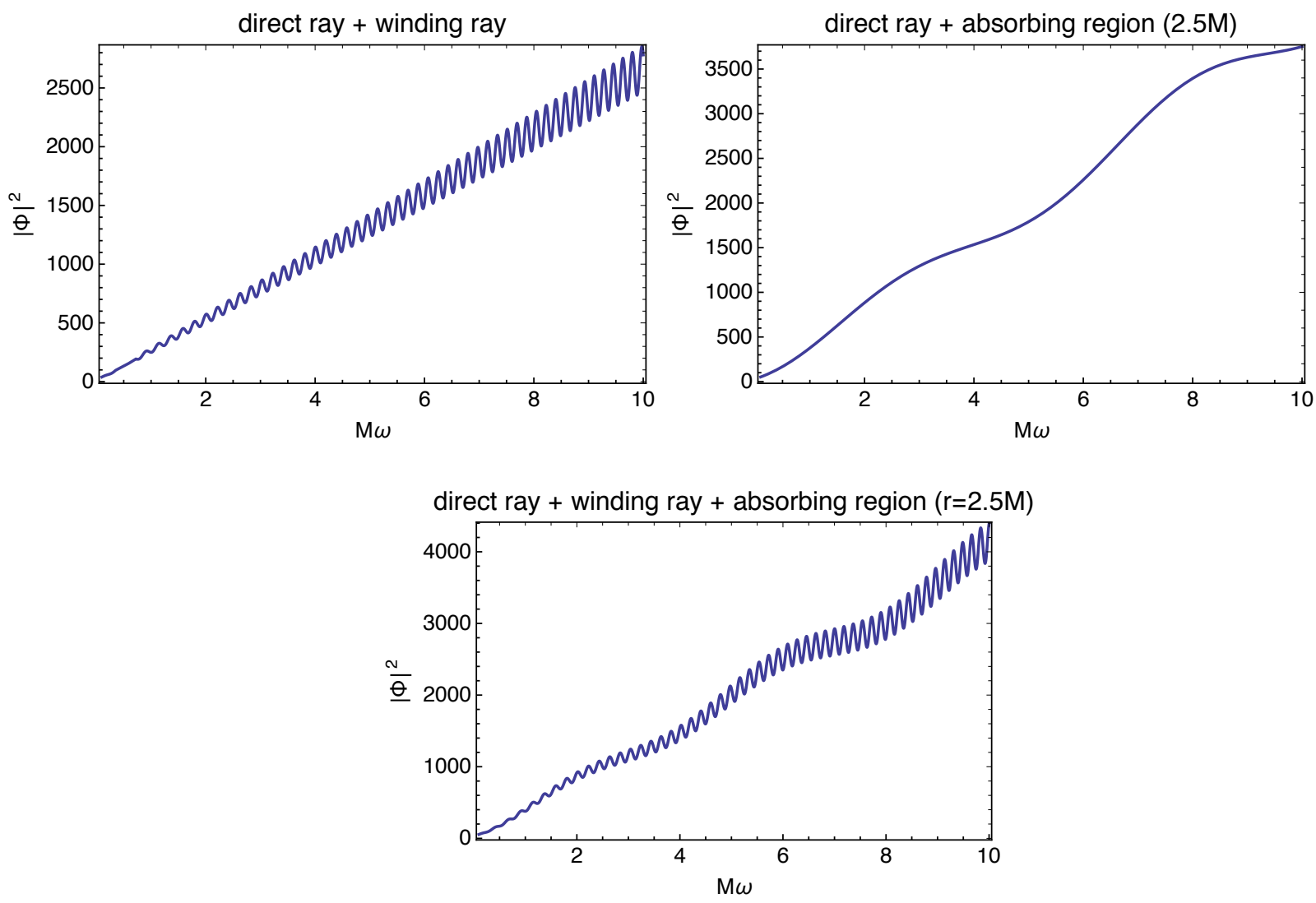

FIG. 15: Effect of interference and diffraction in power spectrums. The first panel is the power spectrum obtained by taking into account the interference between direct rays and winding rays. The second panel is the power spectrum obtained by taking into account the diffraction effect for direct rays. The third panel is the power spectrum obtained by taking into account all effects. We assume $b_{0}=2.5 M$ and $b_{c}=3 \sqrt{3} M$ in these plots.

The analytic formula (37) well reproduces behaviors of the power spectrum obtained by our numerical calculation. We can estimate the period of oscillations in the power spectrum. Using (37) and (39), the period of oscillation due to the diffraction effect is

$$
M \Delta \omega_{2} \sim 2 \pi\left(2-\frac{b_{0}^{2}}{2 M \bar{r}}\right)^{-1} \sim 4.8 \quad\left(\text { for } b_{0}=2.5 M, \bar{r}=4.6 M\right) .
$$


Although this value is about 2 times larger than the value obtained by the numerical calculation, the formula shows an increase of period for the larger size of the diffraction region and qualitatively explains the behavior of the power spectrum obtained by numerical calculations. The period of the oscillation due to interference between direct rays and winding rays is

$$
M \Delta \omega_{1} \sim \frac{M}{b_{c}-M / \pi} \sim 0.2 \quad\left(\text { for } b_{c}=3 \sqrt{3} M\right) .
$$

These values are consistent with the period of oscillations in the power spectrums obtained by the numerical calculation for the black hole and stars with smaller radius than the photon sphere.

\section{SUMMARY AND CONCLUSION}

By solving the scalar wave equation numerically, we obtained the scattering wave by the Schwarzschild black hole, the spherical star with a perfect absorbing surface, and the Ellis wormhole, and then we investigated the wave pattern and the power spectrums. We focused on the case that an observer is located at the forward position where we do not expect the interference between direct rays due to the difference of paths lengths in the geometrical optics point of view. Even in this case, we found two kinds of oscillations in the power spectrums. When a gravitating object has the photon sphere (black hole, star

with $r_{\text {star }} \leq 3 M$ and Ellis wormholes), we can see the oscillation of the power spectrum reflecting the interference between the direct ray and the winding ray. Moreover, diffraction effects due to the absorption boundary condition were observed for stars and massive Ellis wormholes. We have justified the periods of these oscillations by analytic evaluation of the wave scattering. As expected, it is possible to distinguish black holes from their mimickers by looking inside of the photon sphere using waves although we cannot tell the difference in the geometrical optics for the present source-object-observer configuration.

As other interesting models, we will consider stars with an internal structure or a reflecting surface and wormholes with a double-peak effective potential [37], which may give echoes in the power spectrums of the scattered waves.

\section{Acknowledgments}

Y. N. was supported in part by JSPS KAKENHI Grant Number $15 \mathrm{~K} 05073$ and S. N. was supported by JSPS KAKENHI Grant No. 17J10770.

\section{Appendix A: Wormhole spacetimes}

As an example of spacetimes with the photon unstable orbit without horizon, we consider the Ellis wormhole [36]. Although the wormhole spacetimes are unstable and may not be realized in our universe, we use them as benchmark models to detect wave optical effects for compact gravitating objects.

The Ellis wormhole spacetime is obtained as the solution of the Einstein-scalar system

$$
R_{\mu \nu}=2 \chi_{, \mu} \chi_{, \nu}, \quad \square \chi=0 .
$$


The metric is given by 36 ]

$$
\begin{aligned}
& d s^{2}=-f d t^{2}+\frac{1}{f}\left(d x^{2}+\left(x^{2}+a^{2}-m^{2}\right) d \Omega^{2}\right), \quad-\infty<x<+\infty \\
& f=\exp \left(-\frac{2 m \chi(x)}{a}\right), \quad \chi(x)=\frac{a}{\sqrt{a^{2}-m^{2}}}\left(\frac{\pi}{2}-\arctan \frac{x}{\sqrt{a^{2}-m^{2}}}\right),
\end{aligned}
$$

where $a$ and $m$ are constants representing the throat size and the mass of the wormhole, respectively. The range of the scalar field is $0(x=+\infty) \leq \chi \leq \frac{\pi a}{\sqrt{a^{2}-m^{2}}}(x=-\infty)$. By introducing a new radial coordinate corresponding to the circumference radius

$$
r=\frac{\sqrt{x^{2}+a^{2}-m^{2}}}{f^{1 / 2}}
$$

the metric becomes

$$
d s^{2}=-f d t^{2}+\frac{d r^{2}}{h}+r^{2} d \Omega^{2}, \quad h=f\left(\frac{d r}{d x}\right)^{2} .
$$

In terms of the scalar field $\chi$,

$$
\begin{aligned}
& x=\sqrt{a^{2}-m^{2}} \cot \left(\frac{\sqrt{a^{2}-m^{2}}}{a} \chi\right), \\
& r=\frac{\sqrt{a^{2}-m^{2}} e^{m \chi / a}}{\sin \left(\frac{\sqrt{a^{2}-m^{2}}}{a} \chi\right)}, \quad r_{\min }=a \exp \left[\frac{m}{\sqrt{a^{2}-m^{2}}} \operatorname{Arccot}\left(\frac{m}{\sqrt{a^{2}-m^{2}}}\right)\right], \\
& h=\left[\cos \left(\frac{\sqrt{a^{2}-m^{2}}}{a} \chi\right)-\frac{m}{\sqrt{a^{2}-m^{2}}} \sin \left(\frac{\sqrt{a^{2}-m^{2}}}{a} \chi\right)\right]^{2} .
\end{aligned}
$$

$r_{\text {min }}$ is the circumference radius of the wormhole throat (at $r=m$ ). The asymptotic behavior of the metric A5 for $x \rightarrow \infty(\chi \rightarrow 0)$ is

$$
\begin{aligned}
& x \sim \frac{a}{\chi}, \quad R \sim \frac{a}{\chi} \sim x, \\
& h \sim 1-\frac{2 m}{x}-\frac{a^{2}-2 m^{2}}{x^{2}}, \quad f \sim 1-\frac{2 m \chi}{a} \sim 1-\frac{2 m}{x} .
\end{aligned}
$$

Thus the metric for $x \rightarrow \infty$ becomes

$$
d s^{2} \approx-\left(1-\frac{2 m}{r}\right) d t^{2}+\left(1+\frac{2 m}{r}+\frac{a^{2}+2 m^{2}}{r^{2}}\right) d r^{2}+r^{2} d \Omega^{2} .
$$

On the other hand, the asymptotic behavior of the metric for $x \rightarrow-\infty\left(\chi \rightarrow \frac{\pi a}{\sqrt{a^{2}-m^{2}}}\right)$ is

$$
\begin{aligned}
& x \sim \frac{-1}{\frac{\pi}{\sqrt{a^{2}-m^{2}}}-\frac{\chi}{a}}, \quad r \sim-x e^{\frac{\pi m}{\sqrt{a^{2}-m^{2}}}}, \quad h \sim 1-\frac{2 m}{x} \sim 1+\frac{2 m e^{\frac{\pi m}{\sqrt{a^{2}-m^{2}}}}}{r}, \\
& f \sim e^{-\frac{2 \pi m}{\sqrt{a^{2}-m^{2}}}}\left(1-\frac{2 m}{x}\right) \sim e^{-\frac{2 \pi m}{\sqrt{a^{2}-m^{2}}}}\left(1+\frac{2 m e^{\frac{\pi m}{\sqrt{a^{2}-m^{2}}}}}{r}\right) .
\end{aligned}
$$


Thus the metric for $x \rightarrow-\infty$ represents a gravitating object with negative mass $-m e^{\frac{\pi m}{\sqrt{a^{2}-m^{2}}}}$. For massless case $m=0$, the metric reduces to

$$
d s^{2}=-d t^{2}+d x^{2}+\left(x^{2}+a^{2}\right) d \Omega^{2} .
$$

In this case, the gravitational potential in the weak field region behaves as $\propto r^{-2}$ and the law of gravity is different from that for a point mass.

The shape of the effective potential for the wormhole is shown in Fig. 16.

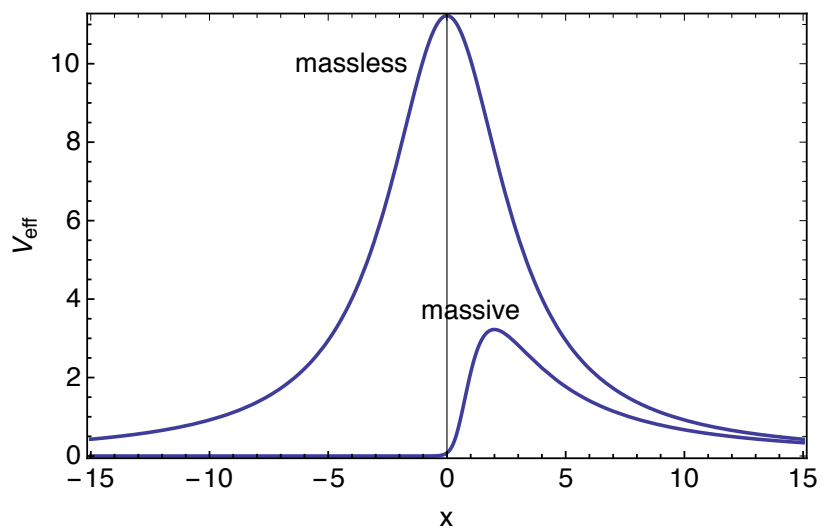

FIG. 16: Effective potentials for the Ellis wormhole $(\ell=10)$ for the massless case $(a=3 M, m=0)$ and the massive case $(a=1.305716 M, m=M)$. The throat of the wormhole is located at $x=m$ and the peak of the potential in the eikonal limit $\ell \gg 1$ is at $x \approx 2 m$. This place corresponds to the photon sphere.

In our numerical calculations, we adopt the wormhole parameters as $a=3 M, m=0$ (massless case) and $a=1.305716 M, m=M$ (massive case). The circumference radii of the throat for both wormholes are $3 M$ and the circumference radii of the photo sphere are $3 M$ (massless case) and 3.4823M (massive case).

[1] T. E. H. T. Collaboration, "First M87 Event Horizon Telescope Results. I. The Shadow of the Supermassive Black Hole", The Astrophys. J. Lett. 875, (2019) 875:L1.

[2] P. V. P. Cunha, C. A. R. Herdeiro, and E. Radu, "Fundamental photon orbits : Black hole shadows and spacetime instabilities", Phys. Rev. D 96, (2017) 024039.

[3] P. V. P. Cunha, A. Font, C. Herdeiro, E. Radu, N. Sanchis-gual, and M. Zilhão, "Lensing and dynamics of ultracompact bosonic stars", Phys. Rev. D 96, (2017) 104040.

[4] P. V. P. Cunha, E. Berti, and C. A. R. Herdeiro, "Light-Ring Stability for Ultracompact Objects", Phys. Rev. Lett. 119, (2017) 251102.

[5] P. V. P. Cunha, C. A. R. Herdeiro, and P. V. P. Cunha, "Shadows and strong gravitational lensing : a brief review", Gen. Relativ. Gravit. 50, (2018) 1-27.

[6] P. V. P. Cunha, C. A. R. Herdeiro, and M. J. Rodriguez, "Shadows of exact binary black holes", Phys. Rev. D 98, (2018) 44053.

[7] P. V. P. Cunha, C. A. R. Herdeiro, and M. J. Rodriguez, "Does the black hole shadow probe the event horizon geometry?", Phys. Rev. D 97, (2018) 84020. 
[8] N. Sakai, "Gravastar shadows", Phys. Rev. D 90, (2014) 104013.

[9] T. Ohgami and N. Sakai, "Wormhole shadows", Phys. Rev. D 91, (2015) 124020.

[10] T. Ohgami and N. Sakai, "Wormhole shadows in rotating dust", Phys. Rev. D 94, (2016) 064071.

[11] V. Cardoso, A. Miranda, E. Berti, H. Witek, and V. Zanchin, "Geodesic stability, Lyapunov exponents, and quasinormal modes", Phys. Rev. D 79, (2009) 064016.

[12] N. Andersson and B. Jensen, "Scattering by black holes", arXiv:gr-qc/0011025.

[13] K. Glampedakis and N. Andersson, "Scattering of scalar waves by rotating black holes", Class. Quantum Grav. 18, (2001) 1939-1966.

[14] K.-i. Kanai and Y. Nambu, "Viewing black holes by waves", Class. Quantum Grav. 30, (2013) 175002.

[15] Y. Nambu and S. Noda, "Wave optics in black hole spacetimes: the Schwarzschild case", Class. Quantum Grav. 33, (2016) 075011.

[16] R. A. Matzner, "Scattering of massless scalar waves by a Schwarzschild " singularity"", J. Math. Phys. 9, (1968) 163-170.

[17] N. Sánchez, "Elastic scattering of waves by a black hole", Phys. Rev. D 18, (1978) 1798-1804.

[18] F. A. Handler and R. A. Matzner, "Gravitational wave scattering", Phys. Rev. D 22, (1980) 2331-2348.

[19] T.-r. Zhang and C. Dewitt-morette, "WKB Cross Section for Polarized Glories of Massless Waves in Curved Space-Times.", Phys. Rev. Lett. 52, (1984) 2313.

[20] R. A. Matzner, C. Dewitte-morette, and B. Nelson, "Glory scattering by black holes", Phys. Rev. D 31, (1985) 1869-1878.

[21] J. Futterman, F. Handler and R. A. Matzner, Scattering from Black Holes (Cambridge Monographs on Mathematical Physics). (1988).

[22] S. R. Dolan, "Scattering of long-wavelength gravitational waves", Phys. Rev. D 77, (2008) 044004 .

[23] S. R. Dolan, "Scattering and absorption of gravitational plane waves by rotating black holes", Class. Quantum Grav. 25 (2008) 235002.

[24] L. C. Crispino, S. R. Dolan, and E. S. Oliveira, "Scattering of massless scalar waves by Reissner-Nordström black holes", Phys. Rev. D 79 (2009) 064022.

[25] L. C. Crispino, S. R. Dolan, A. Higuchi, and E. S. De Oliveira, "Inferring black hole charge from backscattered electromagnetic radiation", Phys. Rev. D 90, (2014) 1-5.

[26] L. C. Crispino, S. R. Dolan, A. Higuchi, and E. S. De Oliveira, "Scattering from charged black holes and supergravity", Phys. Rev. D 92, (2015) 1-5.

[27] L. C. S. Leite, S. R. Dolan, and L. C. B. Crispino, "Absorption of electromagnetic and gravitational waves by Kerr black holes: Shadows, superradiance and the spin-helicity effect", Phys. Lett. B 774, (2017) 1-5.

[28] J. Alexandre and K. Clough, "Black hole interference patterns in flavor oscillations", Phys. Rev. D 98 (2018) 043004.

[29] C. A. Sporea, "MOG black hole scattering", arXiv:1812.09945 .

[30] S. R. Dolan and T. Stratton, "Rainbow scattering in the gravitational field of a compact object", Phys. Rev. D 95, (2017) 1-20.

[31] T. Stratton and S. R. Dolan, "Rainbow scattering of gravitational plane waves by a compact body", arXiv:1903.00025.

[32] I. I. Cotăescu and C. A. Sporea, "Scattering of Dirac fermions by spherical massive bodies", Eur. Phys. J. C 79, (2019) 1-8. 
[33] C.-M. Yoo, T. Harada, and N. Tsukamoto, "Wave effect in gravitational lensing by the Ellis wormhole", Phys. Rev. D 87, (2013) 084045.

[34] K. Nakajima and H. Asada, "Deflection angle of light in an Ellis wormhole geometry", Phys. Rev. D 85, (2012) 107501.

[35] K. Sharma, Optics: principles and applications (Academic Press, Tokyo, 2006).

[36] H. G. Ellis, "Ether flow through a drainhole: A particle model in general relativity", J. Math. Phys. 14, (1973) 104-118.

[37] P. Bueno, P. A. Cano, F. Goelen, T. Hertog and B. Vercnocke, "Echoes of Kerr-like wormholes", Phys. Rev. D 97, (2018) 024040. 